\section{Long-term effect of phacoemulsification on trabeculectomy function}

A Longo, MG Uva, A Reibaldi, T Avitabile

A Longo, MG U
and M Reibaldi

\begin{abstract}
Purpose To investigate the long-term effect of phacoemulsification on intraocular pressure (IOP) in trabeculectomized eyes and to identify the factors affecting the IOP changes. Methods In consecutive patients with previous trabeculectomy (TE) who underwent uncomplicated clear cornea phacoemulsification we evaluated intraocular pressure, need of antiglaucomatous medical therapy or glaucoma surgery, and length of glaucoma control without therapy. Glaucoma therapy was prescribed in the presence of IOP $>18 \mathrm{~mm} \mathrm{Hg}$ or worsening of the visual field. A group of trabeculectomized eyes that did not receive cataract surgery was retrospectively selected as a control. Multivariate analysis was used to test factors
\end{abstract} related to final outcome of the treatment. Results One hundred and eight eyes with previous TE that received phacoemulsification and 108 controls were included in the study. Phacoemulsification was performed $60 \pm 21$ months after TE. After a mean follow-up of $66 \pm 28$ months, mean IOP was significantly increased in the cataract surgery group (by $1.7 \pm 4.3 \mathrm{~mm} \mathrm{Hg}$ ) and in the control group (by $2.3 \pm 4.3 \mathrm{~mm} \mathrm{Hg}$ )(both $P<0.001$ ); in two groups, respectively, 31 eyes $(28.7 \%)$ and 17 eyes $(15.7 \%)$ had received glaucoma therapy (chi-square $P=0.030$ ). Factors related to success (no need of therapy) were use of mitomycin-C (MMC) in previous TE $(P<0.001)$, longer time from TE to cataract surgery $(P=0.007)$, higher preoperative bleb score $(P=0.021)$, and lower baseline IOP $(P=0.042)$.

Conclusions Cataract surgery reduces the function of filtering bleb in some eyes. Factors related to low rate of failure are the previous use of MMC during TE, longer time from TE to cataract surgery, and good preoperative aspect of the bleb. Eye (2015) 29, 1347-1352; doi:10.1038/eye.2015.108; published online 17 July 2015

\section{Introduction}

Although several new surgical techniques have been developed for the treatment of glaucoma, trabeculectomy (TE) is still the most performed intervention.

Cataract surgery can affect the function of the filtering bleb; 2 years after phacoemulsification, an increase of intraocular pressure (IOP), with need of topical medical therapy, has been reported in $29-39 \%$ of the untreated eyes. ${ }^{1-6}$ However, a study showed that the rate of failure of TE after cataract surgery is not different from the natural course of TE. ${ }^{7}$

The use of antimetabolites (AM) during TE reduced the failure rate ${ }^{8-10}$ also, the time between TE and cataract surgery ${ }^{10,11}$ and baseline $\mathrm{IOP}^{7}$ have been identified as factors affecting the outcome.

Most of the studies reported medium-term results (2-3 years follow-up); in the only longterm study, clear cornea phacoemulsification did not affect the survival of TE at 6 and 9 years. ${ }^{12}$

Aim of this study was to evaluate the longterm control of glaucoma in trabeculectomized eyes that underwent phacoemulsification and to identify the factors affecting the IOP changes.

\section{Materials and methods}

In a prospective study, performed at the Eye Clinic of the University of Catania, Italy, from 2005 to 2013, were included all consecutive patients with previous TE who underwent cataract surgery. The study adhered to the tenets of declaration of Helsinki, and it was approved by the local institutional review board; patients gave written informed consent before inclusion in the study.

Inclusion criteria were previous TE performed at least 1 year before for open angle glaucoma, glaucoma control without topical therapy (IOP $<18 \mathrm{~mm} \mathrm{Hg}$ ), and refraction between $+3 \mathrm{D}$ and $-5 \mathrm{D}$.
Eye Clinic, University of Catania, Catania, Italy

Correspondence: A Longo, Eye Clinic, University of Catania, Via Santa Sofia 78, 95123 Catania, Italy Tel: +39095 3781291; Fax: +39095 3781288 . E-mail: ant-longo@libero.it

Received: 22 November 2014

Accepted in revised form: 7 May 2015

Published online:

17 July 2015 
TE technique included the preparation of a superior limbus-based conjunctival flap, the dissection of a halfthickness square scleral flap $\left(\sim 4 \times 4 \mathrm{~mm}^{2}\right)$, followed, if indicated, by the application of mitomycin-C (MMC) over the scleral flap $(0.2 \mathrm{mg} / \mathrm{ml}$ for $2 \mathrm{~min}$ or $0.4 \mathrm{mg} / \mathrm{ml}$ for $4 \mathrm{~min}$ ), then the TE and the basal iridectomy. The scleral flap was sutured with five 10-0 nylon sutures, and Tenon and conjunctiva with a continuous 8-0 vicryl suture. Postoperative management included laser suture lysis, needling, and digital ocular massage.

Patients who had received TE for neovascular, uveitic, or other secondary glaucoma, and patients with previous ocular trauma or posterior segment surgery were excluded from this study. In addition, patients with normal tension glaucoma and patients with high myopia (axial length $>25.5 \mathrm{~mm}$ ) were excluded.

All patients received an uncomplicated clear cornea phacoemulsification (Cat+TE group), performed in topical anesthesia in a site different from the filtering bleb, with stop and chop technique and foldable intraocular lens implantation in the capsular bag. Patients who had a major complication during cataract surgery (posterior capsule rupture with vitreous loss) were excluded. No 5-fluorouracil (5-FU) or MMC application was performed during cataract surgery.

Planned examinations were performed before the cataract surgery, and, after the surgery, at 1 month, at every 3 months in the first year, and at every 6 months in the following years; additional visits were performed in relation to glaucoma control. Glaucoma control was evaluated by examination of the following clinical parameters: daily IOP, visual field, cup-to-disc ratio, and retinal nerve fiber layer thickness. IOP was determined by using a Goldmann applanation tonometer; the visual field was tested by automatic computerized perimetry (24-2, SITA-standard, HFA-II, Humphrey Field Analyzer; Carl Zeiss, Jena, Germany); cup-to-disc ratio was assessed at slit lamp with a $+60 \mathrm{D}$ lens; and retinal nerve fiber layer thickness was determined by Stratus OCT (OCT3; ZeissHumphrey, San Leandro, CA, USA). Controls were performed by physicians who were not aware of the aim of the research. The preoperative maximal area and height of the bleb were graded in anterior segment photographs by two independent observers on magnified pictures by using Moorfields classification. ${ }^{13}$ Maximal area values of 0 and $25 \%$ received score 0 , whereas values of $50 \%$ and higher received score 1 ; height values of 1 (flat) and 4 (encapsulated) received score 0 , whereas values of 2 and 3 received score 1 ; the sum of the values of the maximal area and of the height of the bleb was the final bleb score.

After cataract surgery, bleb massage or needling was performed if necessary. In case of IOP $>18 \mathrm{~mm} \mathrm{Hg}$, or worsening of the visual field, antiglaucomatous topical therapy was prescribed, and the patient was considered as a failure.

A group of consecutive trabeculectomized eyes that did not receive cataract surgery was retrospectively selected and used as a control (only-TE group). Controls were matched by the period when TE was performed (to have a similar follow-up) and the use of MMC. The same inclusion criteria (glaucoma control without topical therapy (IOP $<18 \mathrm{~mm} \mathrm{Hg}$ ) and refraction) and exclusion criteria used for the study group were used for the control group.

\section{Statistical analysis}

The values of all parameters in two groups (Cat+TE and only TE) were compared by $t$-test or $\chi^{2}$-test. Survival analysis according to time free of therapy in two groups was performed by using Kaplan-Meier test, and survival rates were compared by using log-rank (Mantel-Cox) test.

To assess factors related to TE failure, we performed a univariate and multivariate analysis based on a Cox proportional hazards regression model (time free from therapy as outcome); this model included gender, age, preoperative IOP, time between TE and cataract surgery, and use of MMC in previous TE. All predictors that had a $P$-value $<0.15$ (cutoff) in the univariate analysis were included in the multivariate analysis.

In Cat+TE group, Kaplan-Meier survival analysis according to time free of therapy was performed in eyes with low (1-2) and high (3-4) bleb score and in eyes that had received TE with MMC or TE without MMC; survival rates were compared by log-rank (Mantel-Cox) test.

$P$-values $<0.05$ were considered as statistically significant.

Table 1 Demographics

\begin{tabular}{llcc}
\hline & & Cat+TE (n=108) & Only TE (n=108) \\
\hline $\begin{array}{llc}\text { Age at cataract surgery } \\
\text { Sex }\end{array}$ & Years & $66 \pm 6(52-79)$ & $58 \pm 5(46-63)$ \\
Time TE-cat. surgery & Months & $61 \mathrm{~m}, 47 \mathrm{f}$ & $63 \mathrm{~m}, 45 \mathrm{f}$ \\
MMC use & Eyes & $60 \pm 21(16-104)$ & $57 \pm 20(14-102)$ \\
& & $58(0.2 \%$ for 2 min $)$ & 71 \\
& & $13(0.4 \%$ for 4 min $)$ & $52(0.2 \%$ for 2 min $)$ \\
\end{tabular}




\section{Results}

One hundred and eight eyes of 108 patients $(61 \mathrm{~m}, 47 \mathrm{f})$ that had received an uncomplicated phacoemulsification (Cat+TE group) were included in the study; mean age at the cataract surgery was $66 \pm 6$ years. Mean time from TE to cataract surgery was $60 \pm 21$ months: 71 eyes had received TE with MMC. Preoperative bleb score was low (1-2) in 36 eyes and high (3-4) in 72 eyes. Mean follow-up after phacoemulsification was $66 \pm 28$ months.

In the control group (only-TE group), mean age at the study entry (corresponding to time of cataract surgery in other group) was $58 \pm 5$ years $(P<0.001$ vs Cat + TE group). Mean time from TE to the study entry was $57 \pm 20$ months. Mean follow-up was $68 \pm 26$ months. (Table 1 )

At last follow-up visit, in Cat+TE group and in only-TE group, mean IOP had significantly increased

(respectively, by $1.7 \pm 4.3 \mathrm{~mm} \mathrm{Hg}$ and by $2.3 \pm 4.3 \mathrm{~mm} \mathrm{Hg}$, both paired $t$-test $P<0.001$ ); in two groups, 31 eyes (28.7\%) and 17 eyes $(15.7 \%)$ had required topical therapy to control glaucoma (chi-square $P=0.033$ ), although no eye had required further glaucoma surgery. (Table 2)

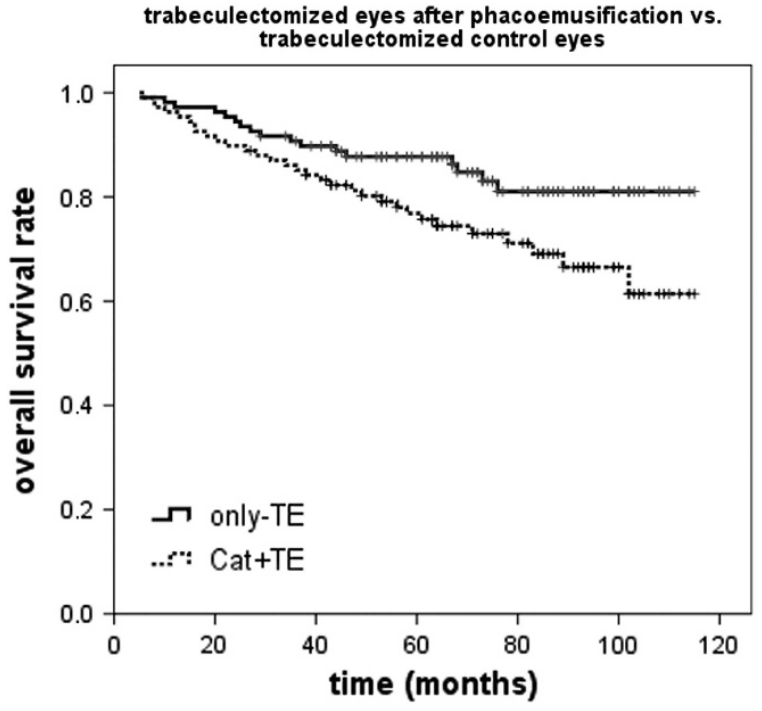

Figure 1 Kaplan-Meier survival analysis of patients with previous TE who received uncomplicated phacoemulsification (Cat+TE) vs trabeculectomized control eyes (only TE). The outcome was the time free of therapy. The log-rank test showed a significant difference between the two groups $(P=0.030)$.

Table 2 Intraocular pressure and outcome in trabeculectomized eyes that received uncomplicated phacoemulsification (Cat+TE) and in trabeculectomized control eyes (Only TE)

\begin{tabular}{|c|c|c|c|c|c|}
\hline & & Cat+TE $(\mathrm{n}=108)$ & Only TE $(\mathrm{n}=108)$ & P-value (t-test) & P-value $\left(\chi^{2}\right)$ \\
\hline Preoperative IOP & $\mathrm{mm} \mathrm{Hg}$ & $14.7 \pm 2.4$ & $14.5 \pm 2.3$ & 0.486 & \\
\hline Last IOP & $\mathrm{mm} \mathrm{Hg}$ & $16.4 \pm 4.1$ & $16.8 \pm 4.0$ & 0.465 & \\
\hline IOP change & $\mathrm{mm} \mathrm{Hg}$ & $+1.7 \pm 4.3$ & $+2.3 \pm 4.3$ & 0.284 & \\
\hline Bleb needling & Eyes & $42(38.9 \%)$ & $26(24.1 \%)$ & & 0.028 \\
\hline Therapy & Eyes & $31(28.7 \%)$ & $17(15.7 \%)$ & & 0.033 \\
\hline
\end{tabular}
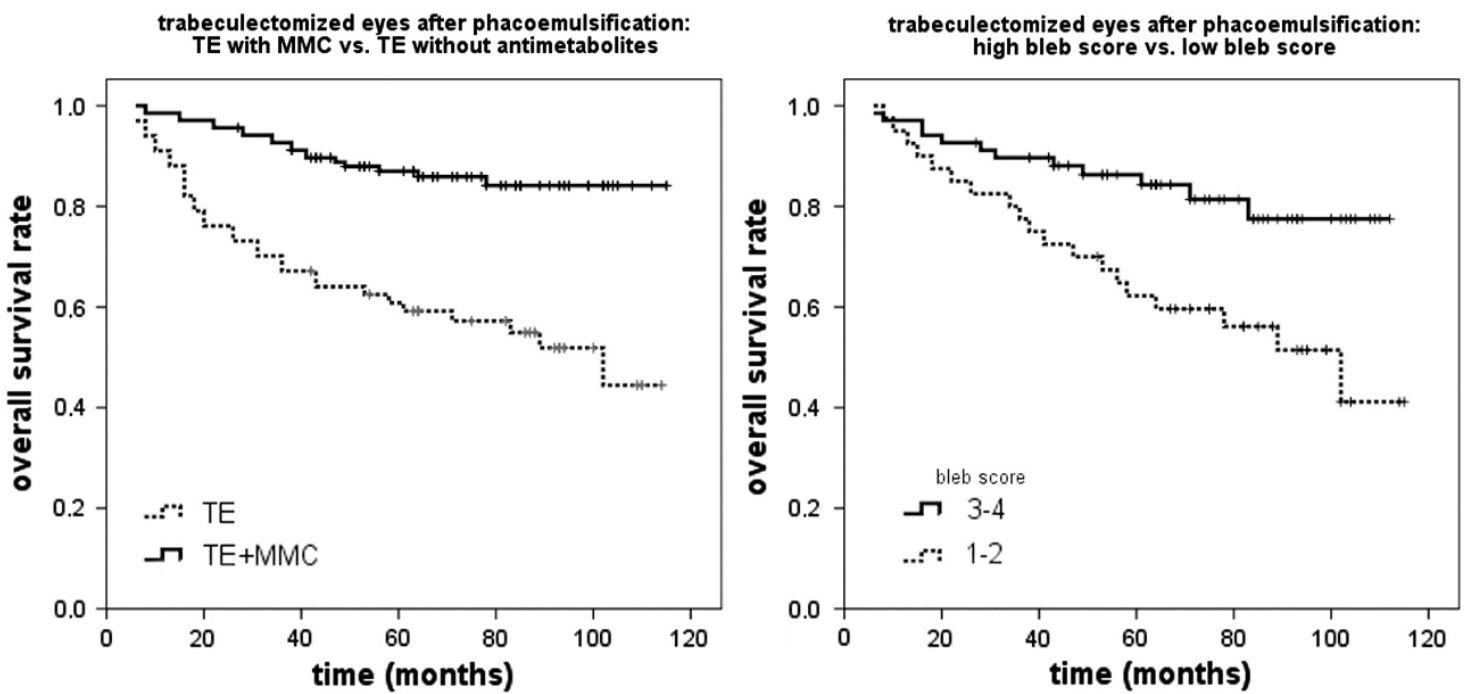

Figure 2 Kaplan-Meier survival analyses of patients with previous TE who received uncomplicated phacoemulsification (Cat+TE). Survival analyses for TE with MMC (TE+MMC) vs TE without AM (TE) (left), and high bleb score (3-4) vs low bleb score (1-2) (right). The outcome was the time free of therapy. The log-rank test showed significant differences in both parameters investigated $(P=0.005$ and $P<0.001$, respectively). 
Figure 1 shows the results of Kaplan-Meier survival analyses according to time free of therapy in the two groups. The estimated success rates (SE, with 95\% CI) were 90.69 (3.71) (CI 83.41-97.97) for Cat+TE eyes and 101.19 (3.09) (CI 95.14-107.24) for only-TE eyes (log-rank test $P=0.030)$.

Multivariate analysis showed that factors related to the success (no need of topical therapy) were use of MMC during the previous TE $(P<0.001)$, longer time from TE to cataract surgery $(P=0.007)$, higher bleb score $(P=0.021)$, and lower baseline IOP $(P=0.042)$.

Figure 2 shows the results of Kaplan-Meier survival analyses performed in the Cat+TE group according to the bleb score and the use of MMC in previous TE. The estimated success rates (SE, with 95\% CI) were as follows:

- 78.41 (6.38) (CI 65.91-90.92) for low bleb score (1-2) and 96.72 (4.02) (CI 88.83-104.60) for high bleb score (3-4);

- 74.37 (5.41) (CI 63.77-84.96) for TE without MMC and 102.95 (2.58) (CI 97.89-108.01) for TE with MMC.

The log-rank test showed a significant difference between the two groups for both parameters investigated $(P=0.005$ and $P<0.001$, respectively).

\section{Discussion}

In this study, with a mean follow-up of 66 months after an uncomplicated phacoemulsification, we found that $28.7 \%$ of the trabeculectomized eyes required topical medical therapy to control glaucoma; in the same period, $15.7 \%$ of the trabeculectomized control eyes received a therapy, suggesting that cataract surgery affects the function of filtering bleb in some eyes.

Other studies have reported, after 2 years of follow-up, a failure (with need of topical medical therapy) in $29-45 \%$ of the eyes treated by a TE without $\mathrm{AM}^{2-7}$ and in $18-24 \%$ of eyes that had received a TE with AM. In controls, after TE with AM, a failure was found in $4-7 \%$ of the eyes (Table 3). ${ }^{8-10}$

In a long-term study, the success rate at 6 and 9 years was 66 and $44 \%$ in eyes with cataract surgery after TE, and 69 and 55\% in controls (only TE); almost half of the eyes (18 of 40) in the two groups received a TE with 5-FU. ${ }^{12}$

The low rate of failure detected in our long-term study could be ascribed to the use of MMC in most of the eyes during TE; multivariate analysis showed that this factor was significantly related to the success.

We also found that a longer time from TE to cataract surgery is related to the success; this result is consistent with the findings of Husain et al, ${ }^{11}$ which found a decrease of failure with increasing time from TE

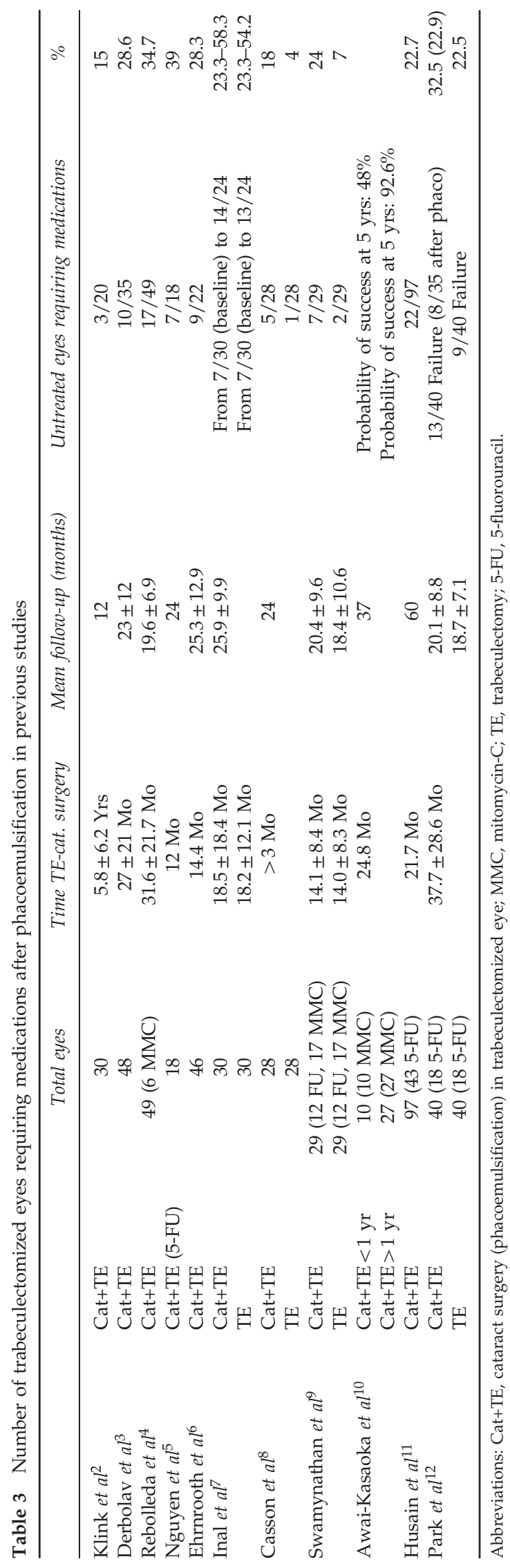


(hazard ratios at 6 months, 1 year, and 2 years were 3.00, 1.73 , and 1.32 , respectively); Awai-Kasaoka et al ${ }^{10}$ found an increased risk of failure if phacoemulsification was performed within 1 year after TE, and suggested that TE could require 1 or 2 years to be stabilized against phacoemulsification.

After phacoemulsification a reduction of the size and the height of the bleb can occur. ${ }^{4,7,14}$ Failure of TE has been ascribed mainly to anterior chamber inflammation after surgery leading to fibrosis, ${ }^{4,15-17}$ through an upregulation of the fibrogenic cytokines; $;^{18}$ an increased number of conjunctival fibroblasts and inflammatory cells has been found. ${ }^{19,20}$ An increased survival of the bleb function has been obtained by subconjunctival injections of 5-FU, performed at the end of phacoemulsification (rate of failure: 13.6 vs $36.4 \%$ ), ${ }^{15}$ or in early postoperative period (rate of failure 4.0 vs $16.7 \% \mathrm{P}=0.78$ ). ${ }^{21}$

Our results show that preoperative bleb morphology is related to long-term outcome: by UBM, Wang et al ${ }^{22}$ found that the preoperative visibility of route under the scleral flap and the reflectivity inside the bleb were significantly associated with success. Bleb needling, or subconjunctival 5-FU injection, could be hypothesized before cataract surgery in eyes with low score bleb (small, flat, or encapsulated) to improve bleb function and to reduce preoperative IOP. We found that a higher preoperative IOP is related to failure, consistently with results of Rebolleda et $a l^{4}$ which found an association of failure with preoperative IOP $>10 \mathrm{~mm} \mathrm{Hg}$, and of Inal et al, ${ }^{7}$ which reported an association with IOP and number of therapies.

This study has several limitations, mainly that cases had variable follow-up, and the two groups had different ages. However, all patients were followed at one Center, and the same treatment strategies were used during follow-up period.

In conclusion, phacoemulsification reduces the function of $\mathrm{TE}$ in some eyes. Factors related to low rate of failure are the previous use of $\mathrm{MMC}$ during $\mathrm{TE}$, longer time from TE to cataract surgery, and good preoperative aspect of the bleb.

\section{Summary}

\section{What was known before}

- Cataract surgery may decrease the function of trabeculectomy. Studies with 2-3 years follow-up reported a need of medication in 18-39\% of the trabeculectomized eyes after uncomplicated phacoemulsification.

\section{What this study adds}

- At long term (after a mean follow-up of 66 months), 28.7\% of the trabeculectomized eyes required therapy after an uncomplicated phacoemulsification $v$ s $15.7 \%$ of the trabeculectomized control eyes. Use of MMC in previous trabeculectomy, longer time between trabeculectomy and cataract surgery, and a good preoperative aspect of the bleb may preserve the function of the bleb after cataract surgery.

\section{Conflict of interest}

The authors declare no conflict of interest.

\section{References}

1 Patel HY, Danesh-Meyer HV. Incidence and management of cataract after glaucoma surgery. Curr Opin Ophthalmol 2013; 24: 15-20.

2 Klink J, Schmitz B, Lieb WE, Klink T, Grein HJ, Sold-Darseff $\mathrm{J}$ et al. Filtering bleb function after clear cornea phacoemulsification: a prospective study. Br J Ophthalmol 2005; 89: 597-601.

3 Derbolav A, Vass C, Menapace R, Schmetterer K, Wedrich A. Long-term effect of phacoemulsification on intraocular pressure after trabeculectomy. J Cataract Refract Surg 2002; 28: $425-430$.

4 Rebolleda G, Muñoz-Negrete FJ. Phacoemulsification in eyes with functioning filtering blebs: a prospective study. Ophthalmology 2002; 109: 2248-2255.

5 Nguyen DQ, Niyadurupola N, Tapp RJ, O'Connell RA, Coote MA, Crowston JG. Effect of phacoemulsification on trabeculectomy function. Clin Experiment Ophthalmol 2014; 42: 433-439.

6 Ehrnrooth P, Lehto I, Puska P, Laatikainen L. Phacoemulsification in trabeculectomized eyes. Acta Ophthalmol Scand 2005; 83: 561-566.

7 Inal A, Bayraktar S, Inal B, Bayraktar Z, Yilmaz OF. Intraocular pressure control after clear corneal phacoemulsification in eyes with previous trabeculectomy: a controlled study. Acta Ophthalmol Scand 2005; 83: 554-560.

8 Casson R, Rahman R, Salmon JF. Phacoemulsification with intraocular lens implantation after trabeculectomy. J Glaucoma 2002; 11: 429-433.

9 Swamynathan K, Capistrano AP, Cantor LB, WuDunn D. Effect of temporal corneal phacoemulsification on intraocular pressure in eyes with prior trabeculectomy with an antimetabolite. Ophthalmology 2004; 111: 674-678.

10 Awai-Kasaoka N, Inoue T, Takihara Y, Kawaguchi A, Inatani $\mathrm{M}$, Ogata-Iwao $\mathrm{M}$ et al. Impact of phacoemulsification on failure of trabeculectomy with mitomycin-C. J Cataract Refract Surg 2012; 38: 419-424.

11 Husain R, Liang S, Foster PJ, Gazzard G, Bunce C, Chew PT et al. Cataract surgery after trabeculectomy: the effect on trabeculectomy function. Arch Ophthalmol 2012; 130: 165-170.

12 Park HJ, Kwon YH, Weitzman M, Caprioli J. Temporal corneal phacoemulsification in patients with filtered glaucoma. Arch Ophthalmol 1997; 115: 1375-1380.

13 Wells AP, Crowston JG, Marks J, Kirwan JF, Smith G, Clarke JC et al. A pilot study of a system for grading of drainage blebs after glaucoma surgery. J Glaucoma 2004; 13: 454-460.

14 Mietz H, Andresen A, Welsandt G, Krieglstein GK. Effect of cataract surgery on intraocular pressure in eyes with previous trabeculectomy. Graefe's Arch Clin Exp Ophthalmol 2001; 239: 763-769.

15 Sharma TK, Arora S, Corridan PG. Phacoemulsification in patients with previous trabeculectomy: role of 5-flourouracil. Eye 2007; 21: 780-783.

16 Addicks EM, Quigley HA, Green WR, Robin AL. Histologic characteristics of filtering blebs in glaucomatous eyes. Arch Ophthalmol 1983; 101: 795-798. 
17 Khaw PT, Chang L, Wong TT, Mead A, Daniels JT, Cordeiro MF. Modulation of wound healing after glaucoma surgery. Curr Opin Ophthalmol 2001; 12: 143-148.

18 Nishi O, Nishi K, Ohmoto Y. Synthesis of interleukin-1, interleukin-6, and basic fibroblast growth factor by human cataract lens epithelial cells. J Cataract Refract Surg 1996; 22: 852-858.

19 Allen JB, Davidson MG, Naisse MP, Fleisher LN, McGahan MC. The lens influences aqueous humour levels of transforming growth factor-beta 2. Graefe's Arch Clin Exp Ophthalmol 1998; 236: 305-311.
20 Broadway DC, Grierson I, Hitchings RA. Local effects of previous conjunctival incisional surgery and the subsequent outcome of filtration surgery. Am J Ophthalmol 1998; 125: 805-818.

21 Shahid H, Salmon JF. Use of 5-Fluorouracil injections to reduce the risk of trabeculectomy bleb failure after cataract surgery. J Ocul Pharmacol Ther 2010; 26: 119-123.

22 Wang X, Zhang H, Li S, Wang N. The effects of phacoemulsification on intraocular pressure and ultrasound biomicroscopic image of filtering bleb in eyes with cataract and functioning filtering blebs. Eye 2009; 23: 112-116. 